ustekinumab prescriptions that required validation from the inspection service.

Main outcome measures: gender, age, dose, time in treatment, previous use of a biological antiTNF, changes in frequency of administration, induction posology at the beginning.

Statistics: Descriptive analysis of qualitative and quantitative data, unpaired t-test with SPSS 17.0.

Results The review consisted of 57 patients (56.1\% men) with a mean age of 52 (SD 12.9) for men and 43 years old (SD 19.2) for women $(\mathrm{P}=0.05)$. In 9 patients (one woman) the dose used was $90 \mathrm{mg}$. In 35 cases $(61.4 \%)$ the patients received a previous treatment with biological antiTNF and in 12 patients the treatment started every 12 weeks directly (without induction). The physicians changed the frequency in 10 patients (17.5\%): 5 with doses every 16-20 weeks and 5 with interruptions with a mean of 7.6 months.

Currently 11 patients have stopped the treatment and the average time of treatment is 19.3 months (SD 9.9).

Conclusions Ustekinumab was the first-line biological treatment in $38.6 \%$ of patients.

A significant number of patients used $90 \mathrm{mg}$, and it could be interesting to evaluate whether a $45 \mathrm{mg}$ dose would be sufficiently effective to reduce the cost.

No conflict of interest.

\section{OHP-084 WHAT CONCEPTS ARE USED TO DESCRIBE THE COOPERATION MECHANISMS BETWEEN THE HOSPITAL SECTOR AND PRIMARY CARE? ANALYSIS OF TERMINOLOGY}

doi:10.1136/ejhpharm-2013-000276.457

'S Vogler, 'N Zimmermann, ${ }^{1} \mathrm{C}$ Habl, ${ }^{1} \mathrm{C}$ Leopold, ${ }^{1} \mathrm{~K}$ Habimana, ${ }^{2} \mathrm{~A}$ Mantel-

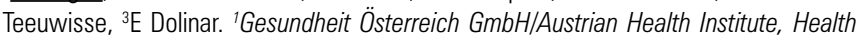
Economics, Vienna, Austria; 2 Utrecht University, WHO Collaborating Centre for Pharmacoepidemiology and Pharmaceutical Policy Analysis, Utrecht, The Netherlands; ${ }^{3}$ Retired, Chief Hospital Pharmacist, Vienna, Austria

Background Cooperation between the hospital sector and primary care is addressed under different names which hampers sharing and identifying existing practises and policies in this field.

Purpose To get a better understanding of the concept of medicines management at the interface of the hospital and primary care sectors (hereafter called interface management).

Materials and Methods Narrative literature review searching Medline, EMBASE, GoogleScholar, Web of Science (ISI), supplemented by hand searching (snowballing) to detect grey literature and contacts with policy makers, researchers and hospital pharmacists to identify further references. Search terms included interface (management), seamless care, continuous care, transitional care, transition in combination with medication, medicines, drugs and pharmaceuticals. Interventions that did not address medicines were excluded; the search period was 1990 to September 2012.

Results In English-language literature, the most commonly applied terms are seamless care, integrated care, comprehensive care, transmural care, transitional care and continuity of care for which, in most cases, generally accepted and repeatedly quoted definitions exist. A more recent terminology is 'interface management'. In many cases, specific projects such as hospital discharge programmes are described without any explicit reference to overall concepts such as interface management or seamless care. Tools such as medicines reconciliation and/or patient counselling can be used to improve medicines management at the interface but they are not necessarily used as specific interface management measures.

Conclusions Even in the English-language literature, the mechanisms of cooperation between the hospital sector and the primary care are referred to under different names. It is recommended to include specific interface management measures as search terms in a literature review on interface management since overall concepts such as seamless care and interface management are likely to yield few results. Terminology work to increase clarity in this field is needed.

No conflict of interest.

\section{Clinical pharmacy and clinical trials (including case series)}

\section{CPC-001 A CLINICAL PHARMACIST FOR OUTPATIENT CONSULTATIONS IN A HEART FAILURE CLINIC}

doi:10.1136/ejhpharm-2013-000276.458

'0 Jullion, ${ }^{2 P}$ Sterckx, ${ }^{2} \mathrm{~S}$ Huez, ${ }^{2} \mathrm{JL}$ Vachiery, ${ }^{1} \mathrm{~S}$ Lorent. 'Erasme Hospital, Pharmacy, Brussels, Belgium; '2Erasme Hospital, Cardiology, Brussels, Belgium

Background Heart Failure (HF) is a severe chronic condition requiring polymedication, which is associated with a risk of nonadherence to chronic Heart Failure (CHF) treatment.

We recently demonstrated that a clinical pharmacist (CP) can be successfully integrated into a cardiology department to improve HF patient care by supervising the treatment. In addition, in 2009 we developed a dedicated CP outpatient consultation integrated in the Heart Failure Clinic (HFC) at our institution.

Purpose To provide a description of the role of the CP in an outpatient HFC.

Materials and Methods 325 patients with HF are monitored at our HFC. All patients are seen by the CP during a dedicated consultation, which includes the following: 1) preparation of the patient's file; 2) the complete history of medical treatment is checked; 3) the record of drugs is updated; 4) vital signs and electrocardiography are performed by the HF nurse. The patient is then seen by the cardiologist who updates the treatment plan. The patient is then seen by the $\mathrm{CP}$ who draws up and issues a plan to put the new treatment into practise, which includes scheduling phone contact for drug uptitration. Finally, the CP and the HF nurse ensure that the plan is followed by weekly scheduled phone consultations.

Results Each HF patient is seen at the outpatient clinic for approximately 45 minutes. On a yearly basis, the CP establishes 584 pharmaceutical plans and performs 197 phone consultations for follow up, resulting in 97 changed treatments.

Conclusions The integration of a CP into the HFC is important to improve management of HF through dedicated outpatient consultations, implementation of treatment plans and checking the patient's adherence.

No conflict of interest.

\section{CPC-002 A MULTICENTRE RETROSPECTIVE STUDY TO EVALUATE THE ECONOMIC IMPACT OF THE PRESCRIBING MODELS FOR TRASTUZUMAB IN THE PIEMONTE REGION}

doi:10.1136/ejhpharm-2013-000276.459

${ }^{1} \mathrm{DA}$ Barilà, ${ }^{1} \mathrm{~A}$ Bianco, ${ }^{2} \mathrm{~S}$ Bustreo, ${ }^{2} \mathrm{M}$ Donadio, ${ }^{3} \mathrm{~S}$ Rosso, ${ }^{1} \mathrm{~F}$ Cattel. ${ }^{1} \mathrm{~A} .0$ Città della Salute e della Scienza di Torino, S.C Pharmacy, Turin, Italy; ${ }^{2}$ A. O Città della Salute e della Scienza di Torino, Medical Oncology Senological Breast Unit, Turin, Italy; ${ }^{3} A .0$ Città della Salute e della Scienza di Torino, CPO Piedmunt, Turin, Italy

Background In recent years, there has been a rapid and constant increase in the costs of cancer treatment but, with limited health care resources, it is essential to consider the economic implications of different health interventions. 
Abstract CPC-002 Table 1 Qualitative evaluation of pharmacoeconomics study

\begin{tabular}{llllll}
\hline $\begin{array}{l}\text { Economics } \\
\text { Evaluation } \\
\text { T in adjuvant }\end{array}$ & $\begin{array}{l}\text { Study } \\
\text { Design }\end{array}$ & $\begin{array}{l}\text { Data } \\
\text { Collection }\end{array}$ & $\begin{array}{l}\text { Analysis and } \\
\text { Interpretation } \\
\text { of results }\end{array}$ & $\begin{array}{l}\text { Final } \\
\text { Score }\end{array}$ & $\begin{array}{l}\text { Total } \\
\text { relative } \\
\text { score }\end{array}$ \\
\hline Blank & $24 / 26$ & $27 / 45$ & $35 / 48$ & 86 & $72.3 \%$ \\
Chen & $23 / 26$ & $30 / 45$ & $37 / 48$ & 90 & $75.6 \%$ \\
Dedes & $24 / 26$ & $31 / 45$ & $35 / 48$ & 90 & $75.6 \%$ \\
Essers & $24 / 26$ & $30 / 45$ & $35 / 48$ & 89 & $74.8 \%$ \\
Garrison & $24 / 26$ & $31 / 45$ & $36 / 48$ & 91 & $76.5 \%$ \\
Kurian & $25 / 26$ & $31 / 45$ & $37 / 48$ & 93 & $78.1 \%$ \\
Liberato & $26 / 26$ & $33 / 45$ & $39 / 48$ & 98 & $82.3 \%$ \\
Lidgren & $26 / 26$ & $34 / 45$ & $38 / 48$ & 98 & $82.3 \%$ \\
Millar & $25 / 26$ & $32 / 45$ & $35 / 48$ & 92 & $77.3 \%$ \\
Neyt & $25 / 26$ & $31 / 45$ & $34 / 48$ & 90 & $75.6 \%$ \\
NICE & $26 / 26$ & $42 / 45$ & $40 / 48$ & 108 & $90.7 \%$ \\
Norum & $25 / 26$ & $28 / 45$ & $34 / 48$ & 87 & $73.1 \%$ \\
Shiroiwa & $26 / 26$ & $31 / 45$ & $38 / 48$ & 95 & $79.8 \%$ \\
Skedgei & $25 / 26$ & $27 / 45$ & $37 / 48$ & 89 & $74.8 \%$ \\
Van Vlaenderen & $24 / 26$ & $31 / 45$ & $36 / 48$ & 91 & $76.5 \%$ \\
T in MBC & & & & & \\
Poncet & $24 / 26$ & $32 / 45$ & $31 / 48$ & 87 & $73.1 \%$ \\
Norum J & $25 / 26$ & $33 / 45$ & $35 / 48$ & 93 & $78.1 \%$ \\
Elkin & $26 / 26$ & $37 / 45$ & $40 / 48$ & 103 & $86.5 \%$ \\
Lidgren & $26 / 26$ & $36 / 45$ & $38 / 48$ & 100 & $84 \%$ \\
NICE (2002) & $26 / 26$ & $42 / 45$ & $40 / 48$ & 108 & $90.7 \%$ \\
\hline
\end{tabular}

Purpose To evaluate the economic impact of the different prescribing models for trastuzumab on overall costs for breast cancer treatments in the Piemonte Region.

Materials and Methods We systematically reviewed the MEDLINE-indexed, English-language literature to identify published, peer-reviewed economic analyses of trastuzumab in HER2 \pm treatment of breast cancer. We rated study quality as per the Drummond criteria.

Direct medical and unit costs were calculated from the perspective of a Regional health care system. We derived patient data by consulting a Regional administrative database and screening by File F File C and SDO for each patient treated in 2010. To obtain valid data, it is necessary to combine the data from this study into a single model, with an epidemiological measure from the Piemonte Cancer Registry. It is recommended to use an empirical Bayesian analysis to conduct this study because there is no single estimator for the parameters.

Results The search strategy identified 948 articles, of which 340 were citations. From the 608 remaining, 23 articles were considered suitable for full review based on the inclusion criteria. Of these, 15 considered adjuvant trastuzumab treatment only, seven examined metastatic breast cancer treatment and one considered treatment with trastuzumab beyond progression. The analysis of the accuracy of information provided by the information systems showed us that there was only $40 \%$ correspondence with the administrative database within Molinette Hospital.

Conclusions Preliminary results confirm the difficulty of obtaining accurate data from the administrative systems. We hope to obtain precise data on trastuzumab prescribing, and thus offer complementary information to cost-effectiveness analysis before the launching of a generic drug.

No conflict of interest.

\section{CPC-003 A RETROSPECTIVE ANALYSIS OF THE SWITCHES FROM ORIGINATOR AND BIOSIMILAR RECOMBINANT HUMAN ERYTHROPOIETINS IN CHRONIC KIDNEY DISEASE}

doi:10.1136/ejhpharm-2013-000276.460

I Uomo, M Pastorello. Provincial Health Unit Palermo, Department of Pharmacy, Palermo, Italy
Background Erythropoiesis stimulating agents (ESAs) has been shown to be highly effective in anaemia in chronic kidney disease (CKD). Various biological ESAs are available such as epoetin alfa, beta, darbepoetin alfa and C.E.R.A, including three biosimilars, epoetin alfa, zeta and theta. National regulations are trying to promote the prescription of the biosimilars, especially in ESA-naive patients. Switching between products is not recommended and the pharmacist can't replace one epoetin with another. However, changes do occur in clinical practise.

Purpose In the Pharmacy Department of the Palermo Local Health Unit (LHU) we observed that nephrologists frequently switch patients but not in order to reduce costs. Therefore, the aim of this study was to calculate the prevalence and patterns of switching and to evaluate the reasons for them and the results for these changes.

Materials and Methods Distributing all the epoetins, after a discharge or a $\mathrm{DH}$ (docetaxel/trastuzumab) regimen and ensuring appropriate continuity of care, the Department collected and retrospectively analysed an electronic database with all the prescriptions for both non-dialysis-dependent CKD or dialysis patients. Furthermore, haemoglobin levels $(\mathrm{Hb})$ were collected, if available, from the paper prescriptions. The period of observations was January 2011June 2012.

Results 2,711 patients received an epoetin for CKD (from a population of 750,550$) .368$ patients $(13.6 \%)$ had been switched. Of this group, only 194 patients were evaluable (98 female, mean age 73.57+-SD:14.21). The inclusion criteria were: receiving ESAs for at least four months; less than 60 days between two prescriptions. Treatments were less commonly switched from biosimilars than originator formulations. Only in 7 cases did nephrologists cite the lack of efficacy of the ESA previously administered, with demonstrated worsening of the patient's clinical status (Aranesp 4, Mircera 2 and NeoRecormon 1). In 9 cases we assumed lack of efficacy of the first ESA, based on measurement of the haemoglobin $(\mathrm{Hb})$ values. In the following prescription the clinicians switched and reported an $\mathrm{Hb}$ level lower than the first $(\leq 10 \mathrm{~g} / \mathrm{L})$. In 24 cases, the ESAs varied with the prescriber. There was no reason for the switch or it was made for trivial reasons. 5 changes from the biosimilar were the pharmacist's wrong decision, due to not checking the patient's last prescription on the database. 5 changes from Mircera occurred after the announcement of a worldwide shortage. Only in 9 cases had the clinicians decided to shift toward a biosimilar on cost grounds. In the remaining cases, $\mathrm{Hb}$ levels remained stable before and after the switch. We can also state that no spontaneous reports of adverse drug reactions regarding ESAs have been received.

Conclusions Our results demonstrates that all the switches were well tolerated. This may support the use of biosimilars in terms of safety and efficacy and switches towards less expensive epoetins. The decision to start ESA treatment with a biosimilar must be considered, and it will also be possible to change pretreated patients.

No conflict of interest.

\section{CPC-004 A REVIEW OF PHARMACISTSÍ INTERVENTIONS IN A NEUROLOGY DEPARTMENT}

doi:10.1136/ejhpharm-2013-000276.461

PA Jolivot, A Gonthier, E Camps, B Bonan. FOCH hospital, Pharmacy, Suresnes, France

Background The adult Department of Neurology is a 42-bed unit that includes an inpatient neurology ward, an inpatient stroke unit and a 10-bed neurological intensive care unit. The computerised physician order entry system available in our hospital is Omnipro. It enables the pharmacy resident to consult the cause of hospitalisation, nurse care, surveillance of medical parameters and to analyse prescriptions.

Purpose To describe the pharmacists' interventions (PIs). 